\title{
Pressure Sensitive Paint Measurements on 15\% Scale Rotor Blades in Hover
}

\author{
Dr. Oliver D. Wong* \\ US Army, Hampton, VA 23681 \\ Dr. A. Neal Watkins ${ }^{\dagger}$ \\ NASA Langley Research Center, Hampton, VA 23681 \\ JoAnne L. Ingram ${ }^{\ddagger}$ \\ Swales Aerospace, Hampton, VA 23681
}

\begin{abstract}
This paper describes a proof of concept test to examine the feasibility of using pressure sensitive paint (PSP) to measure the pressure distributions on a rotor in hover. The test apparatus consisted of the US Army 2-meter Rotor Test Stand (2MRTS) and 15\% scale swept tip rotor blades. Two camera/rotor separations were examined: 0.76 and 1.35 radii. The outer $15 \%$ of each blade was painted with PSP. Intensity and lifetime based PSP measurement techniques were attempted. Data were collected from all blades at thrust coefficients ranging from 0.004 to 0.009 .
\end{abstract}

\section{Nomenclature}

$\alpha \quad=$ Angle of attack, deg

$\mathrm{c}=$ Airfoil chord, $\mathrm{ft}$

$\mathrm{x} / \mathrm{c}=$ Non-dimensional chordwise location

$\mathrm{M} \quad=$ Mach number

$\mathrm{S}_{\mathrm{X}} \quad=$ Horizontal camera, rotor separation distance, $\mathrm{ft}$

$\mathrm{S}_{\mathrm{Y}} \quad=$ Vertical camera, rotor separation distance, $\mathrm{ft}$

I $\quad=$ Wind on intensity

$\mathrm{I}_{\text {Ref }} \quad=$ Reference intensity

$\mathrm{P}=$ Wind on pressure, psi

$\mathrm{P}_{\text {Ref }}=$ Reference pressure, psi

$\mathrm{R}=$ Blade radius, $\mathrm{ft}$

$\mathrm{T}=$ Thrust, $\mathrm{lb}$

$\rho=$ Density, slugs $/ \mathrm{ft}^{3}$

$\Omega \quad=$ Rotational speed, RPM

$\mathrm{C}_{\mathrm{T}} \quad=\quad$ Thrust coefficient, $\frac{T}{\pi R^{2} \rho(\Omega R)^{2}}$

$\mathrm{C}_{\mathrm{P}} \quad=$ Pressure coefficient

\section{Introduction}

Tn order to advance analytical prediction methods used for rotorcraft aerodynamics, acoustics, and interactional 1 effects, it is vital that the fundamental physics of the rotor blade tip flow be better understood. One way to achieve this is to examine the pressure distribution at the blade tip. There has been considerable research involving pressure measurements on rotor blades ${ }^{1-4}$. These measurements, however, typically lack the resolution to capture phenomena such as the nascent tip vortex or dynamic stall. Instrumenting the blades with additional transducers to increase

\footnotetext{
* Research Engineer, RDECOM, AMRDEC, AFDD, JRPO, Mail Stop 286, AIAA Member

${ }^{\dagger}$ Research Chemist, Advanced Sensing \& Optical Measurements Branch, Mail Stop 493, AIAA Member

${ }^{\ddagger}$ Analytical Chemist, Advanced Sensing \& Optical Measurements Branch, Mail Stop 493, AIAA Member
} 
spatial resolution can quickly become prohibitive due to the cost and practicality of fitting a large number of sensors into a small area. The added centrifugal loads of the pressure transducers can rapidly become unmanageable. Pressure sensitive paint (PSP) can potentially overcome the limitations of existing measurement techniques. PSP can offer a significant increase in spatial resolution and a potential cost savings over discrete transducers. Static PSP measurement techniques have matured to the point where they can be used for fixed wing measurements in large scale production wind tunnel environments ${ }^{5-8}$. Dynamic PSP measurement techniques have recently become available. Much research has focused on using dynamic PSP on turbo machinery ${ }^{9-11}$. Dynamic PSP may enable high resolution pressure measurements on helicopter rotors, thus allowing more accurate analytical prediction methods. Work in this area has been very limited to date ${ }^{12-13}$. This paper describes proof of concept measurements using PSP on a rotor blade in hover. These tests were designed to enhance current understanding of the practicality of using dynamic PSP for blade tip pressure measurements in forward flight.

PSP is an optical measurement technique that uses a phenomenon known as oxygen quenched photoluminescence to measure the local oxygen concentration. Luminophores, whose ability to luminesce is inhibited by oxygen, are contained within the paint. Higher local concentrations of oxygen result in lower photoluminescent intensities. Likewise, lower oxygen concentrations result in higher intensities. The Stern-Volmer relationship is used to relate the changes in intensity to pressure. This relationship can be expressed as

$$
\left(\frac{I_{\mathrm{r} \text { e f }}}{I}\right)=A(T)+B(T)\left(\frac{P}{P_{\mathrm{R} \text { e f }}}\right)
$$

where $\mathrm{I}_{\text {Ref }}$ and $\mathrm{P}_{\text {Ref }}$ are the luminescent intensity and static pressure, respectively, obtained at a reference condition. Intensity, I, measured at a desired test condition is then used to calculate pressure, P. The coefficients A and B are temperature dependent values that are typically obtained from laboratory measurements. In order to obtain absolute measurements, the surface temperature must be known to define the coefficients A and B. In general, changes in temperature lead to bias errors in pressure. Because of this, surface pressure measurements using conventional means can be used to "anchor" the pressures through an in situ calibration of the paint.

PSP is typically composed of a binder and luminophores. Since the intensity of the fluorescence is a function of the local oxygen concentration, the binder must be porous enough to allow oxygen to diffuse through the binder and interact with the luminophores. Response time of the luminophores can easily be on the order of sub-milliseconds. The factor limiting PSP frequency response is the binder. The rate at which oxygen can travel through the binder determines the frequency response of the paint. Early binders had low permeability and thus slowed paint response. These were more suitable for measuring static pressures. Recently developed "porous" binders have much higher permeability and increased frequency respons $\mathrm{e}^{14-17}$. Frequency responses on the order of kHz are now possible. For a more in depth review of PSP, see reviews by Hamner ${ }^{18}$ and Liu et al. ${ }^{19}$.

\section{Experimental Setup}

The US Army 2-meter Rotor Test Stand (2MRTS) was used for this test. The 2MRTS is a general research drive system that allows testing of different fuselage configurations. It is powered by a 47 hp water cooled electric motor driving a 5:1 transmission. A five-bladed fully articulated hub was used. One cuff on the hub is instrumented to measure lead-lag and flapping. The 2MRTS can be fitted with two strain gage force balances: one for the rotor loads and one for the fuselage loads. The fuselage balance was not monitored since a fuselage was not used for this test. Additional instrumentation included accelerometers for monitoring system health and an encoder to provide 1/rev and 1024/rev timing pulses. A more thorough review of the 2MRTS can be found in the report by Phelps et al. ${ }^{20}$.

The rotor blades are constructed of carbon fiber, fiberglass and Nomex honeycomb. Blade chord is constant out to the tip, where it is swept and tapered. Blade 1 is instrumented with strain gages to monitor beam-wise and chordwise bending blade moments for safety of flight.

\begin{tabular}{|l|l|}
\hline Rotational Speed (RPM) & 2337 \\
\hline Number of Blades & 5 \\
\hline Blade Chord $(\mathrm{ft})$ & 0.1875 \\
\hline Blade Radius $(\mathrm{ft})$ & 2.96 \\
\hline Tip Speed $(\mathrm{ft} / \mathrm{s})$ & 725 \\
\hline Tip Mach Number & 0.65 \\
\hline
\end{tabular}

Table 1. Rotor Parameters 
All testing was conducted in the Rotor Test Cell (RTC) at the NASA Langley Research Center 14- by 22- Foot Subsonic Tunnel. The RTC is an open room used for propeller static testing and rotor hover testing. Louvers at the bottom and top of the walls are opened to minimize recirculation. The 2MRTS is mounted in the east end of the RTC to a blade strut that places the rotor $9.3 \mathrm{ft}$ above the floor.

A schematic of the experimental setup is shown in Fig. 1. The PSP camera and light emitting diode (LED) light pods were mounted to a tubular aluminum structure. This structure was placed so that its closest member was $7.9 \mathrm{ft}$, or 2.67 rotor radii, from the rotor. The camera and lights were mounted so that the distance between the camera and rotor could be varied both in the horizontal and vertical directions. The top view of the experimental setup is shown in Fig. 2. Also shown in the figure are the two measurement regions. The combinations of separation and measurement location are shown in Table 2.

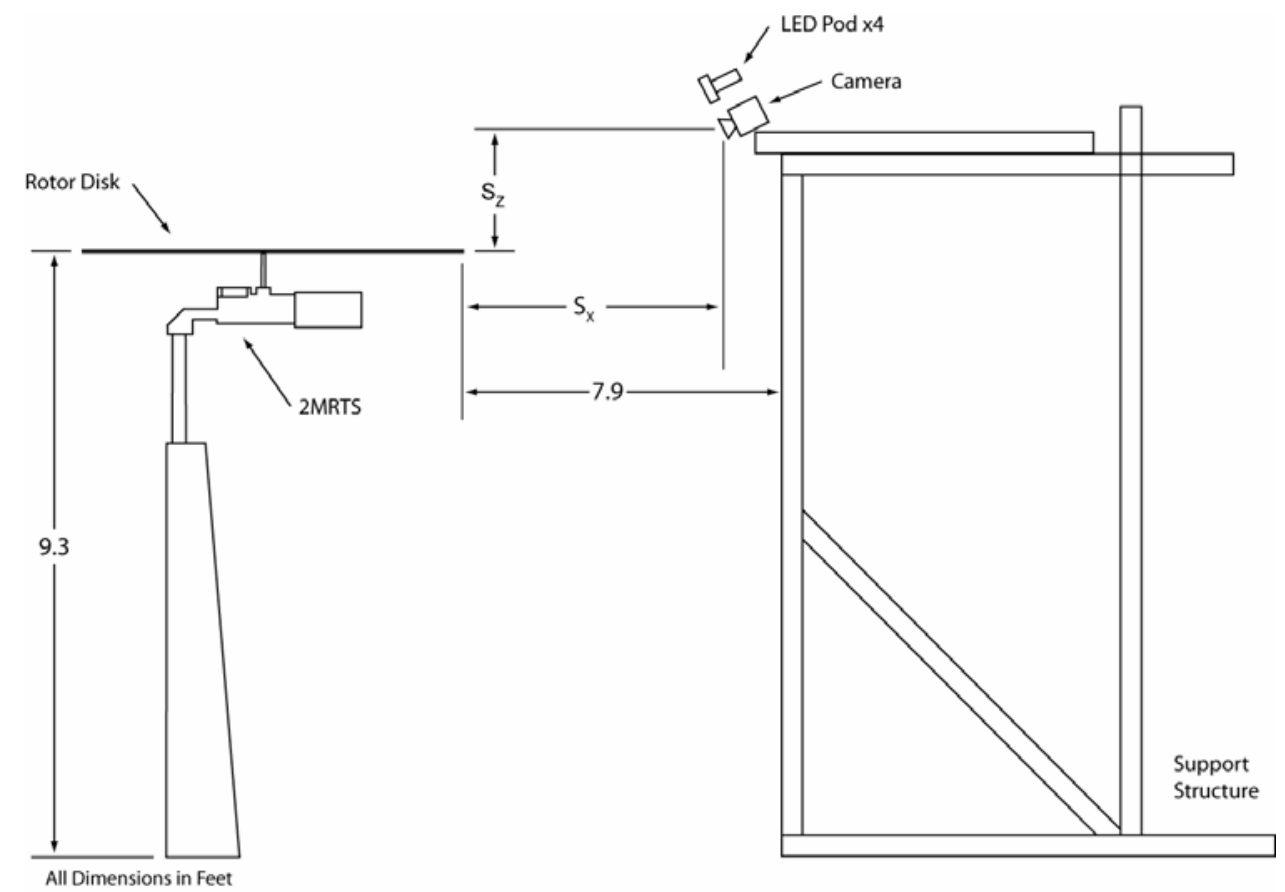

Side View

Figure 1. Experimental Setup
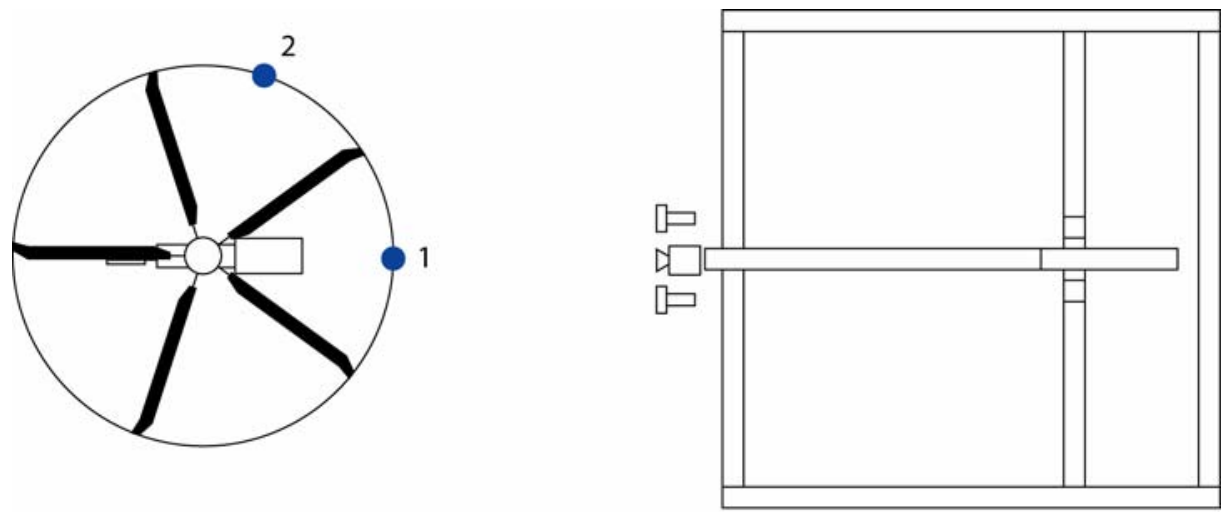

Top View

Figure 2. Experimental setup - location of measurement regions 


\begin{tabular}{|c|c|c|c|}
\hline Configuration & $\begin{array}{c}\text { Horizontal } \\
\text { separation, } \mathrm{S}_{\mathrm{X}} \\
(\mathrm{ft})\end{array}$ & $\begin{array}{c}\text { Vertical } \\
\text { separation, } \mathrm{S}_{\mathrm{Z}} \\
(\mathrm{ft})\end{array}$ & $\begin{array}{c}\text { Measurement } \\
\text { location }\end{array}$ \\
\hline $\mathrm{A}$ & 2.3 & 2.3 & 1 \\
\hline $\mathrm{B}$ & 4.0 & 2.3 & 1 \\
\hline $\mathrm{C}$ & 4.0 & 4.0 & 2 \\
\hline
\end{tabular}

Table 2. Experimental Configurations

The outer 15\% of each blade was painted with a sol-gel based dynamic PSP. Ruthenium bathophenanthroline (Rubath) was used as the luminophore. Its excitation maximum is approximately $450 \mathrm{~nm}$ and maximum emission occurs near $610 \mathrm{~nm}$. The PSP was sprayed on top of a fluoroethylmethacrylate (FEM) polymer primer developed at NASA LaRC. Additionally, each blade was marked with six dots to enable registration between the reference and wind-on images (Fig. 3). The PSP was excited with $460 \mathrm{~nm}$ light from four LED lamps. The dynamic response of the paint was not tested since dynamic pressure fluctuations were not expected in hover. Based upon experiments performed on similar paints at NASA Glenn, the dynamic response of the paint is estimated to be $400 \mathrm{~Hz}^{21}$. All blades were painted to enable examination of blade to blade variation. Although dynamic PSP was used, the flow was assumed to be steady. Dynamic pressure measurements were therefore not acquired. This paint was selected to test its adhesion to the rotor in order to examine the feasibility of using it for future forward flight tests. The life span of the paint was approximately one week. Because of this, it was critical that the data were collected as soon as the blades were painted.

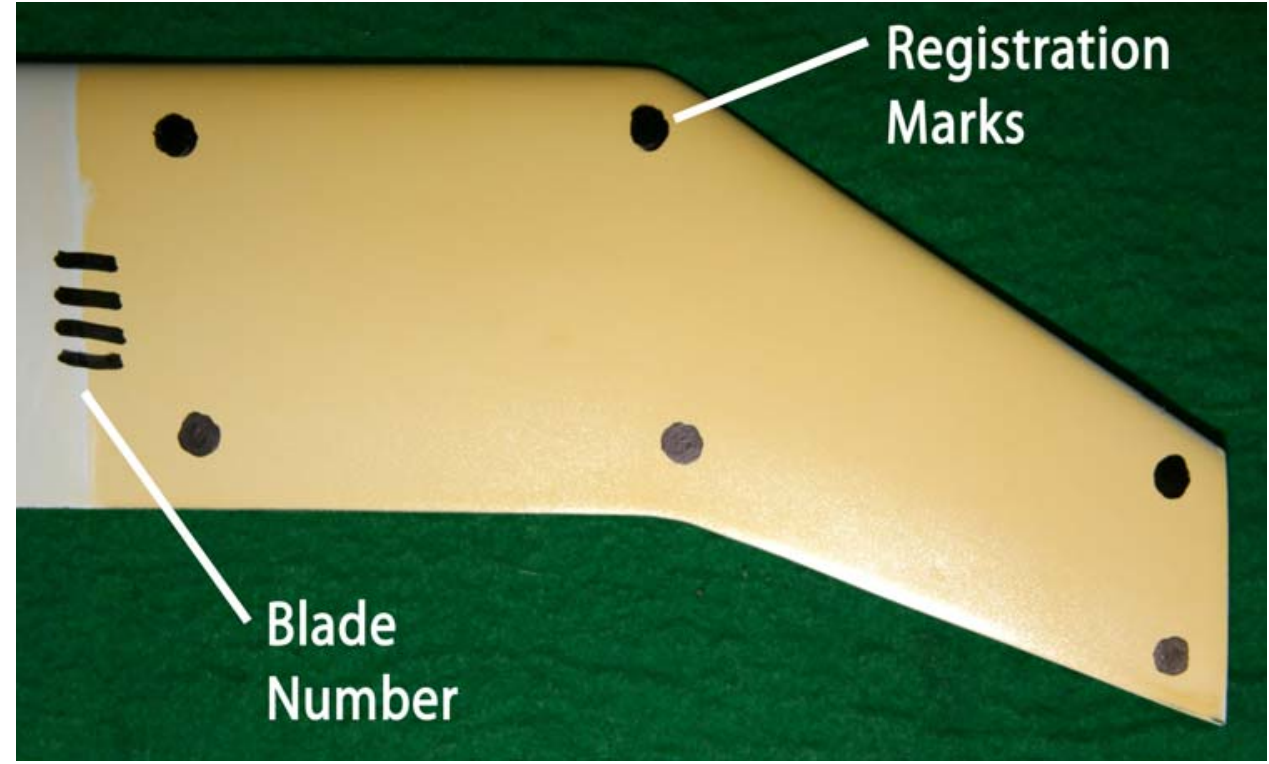

Figure 3. Painted Blade Tip

PSP data were collected during collective sweeps. The sweeps ranged from $6^{\circ}$ to $12^{\circ}$ in $2^{\circ}$ increments. The corresponding thrust coefficients are shown in Table 3.

\begin{tabular}{|c|c|}
\hline $\begin{array}{c}\text { Collective } \\
\text { (deg) }\end{array}$ & $\begin{array}{c}\text { Thrust } \\
\text { Coefficient }\end{array}$ \\
\hline 6 & 0.004 \\
\hline 8 & 0.005 \\
\hline 10 & 0.007 \\
\hline 12 & 0.009 \\
\hline
\end{tabular}

Table 3. Test Conditions

\section{Measurement Techniques}

Intensity and lifetime PSP measurement techniques were tried in these experiments. Both techniques require the ratio of two images to account for inhomogeneities in the lighting and/or paint application. The primary difference 
between the methods is how the reference images are collected. For the intensity based technique, the reference image is collected during wind-off. During each run, the coning of the rotor was noted for each collective setting. Upon completion of the run, the wind off images were obtained by placing the blade at the same coning angle as was noted for each condition.

In the lifetime based technique the reference image is captured when the PSP is being excited, and can be obtained while the rotor is spinning. In the excited state, the sensitivity of the PSP to pressure is negligible and as such can be used for the reference image. Since the reference image and data image can be acquired at the same condition, registration issues between reference and data images are minimized. The downside to this method is that it involves very low light levels. Several hundred LED flashes were required to capture one image. The low light levels involved with the lifetime technique limited the maximum separation between the rotor and the camera and lamps. In Configuration $\mathrm{C}$, which had the maximum separation between the camera and imaging area, the camera was binned to increase its light gathering capability at the expense of spatial resolution. More information on the lifetime techniques used in this work can be found in Watkins et al. ${ }^{22}$

\section{Results \& Discussion}

\section{A. Intensity versus Lifetime Techniques}

Results from the intensity technique suffered from poor registration between the images. The errors introduced by the mis-registration are sufficient to make the data unusable. Even though the blade cuff was positioned identically between the reference and wind-on images, blade flex between the cuff and the tip resulted in significant registration errors. The images collected using the lifetime technique did not suffer from registration errors between images since the reference and wind-on images were taken with the rotor at the desired test condition. The raw images exhibited some blurriness, however. The blurriness was the result of slight variations in blade position due to flapping, leading and lagging of the blade over the several hundred flashes. The registration between images, however, was significantly better than data collected using the intensity method. As a result only measurements using the lifetime technique are presented here.

From the calibration chamber data, the intensity of the paint decreases by approximately $1.5 \%$ for each $1.8^{\circ} \mathrm{F}$ increase. This change in intensity corresponds to an apparent pressure change of approximately 0.3 psi. Given the uncertainty between the ambient temperature and the actual temperature of the blade surface, the data are only being examined in a qualitative manner. Also unaccounted for are temperature gradients across the blade surface. The tip Mach number was subsonic; it was therefore assumed that any temperature gradients on the blade surface were minimal. The ambient temperature change during the runs presented here was $1.9^{\circ} \mathrm{F}$.

\section{B. Results - Thrust Sweeps}

Figure 4 shows the measured surface pressure distribution on Blade 1 at thrust coefficients ranging from 0.004 to 0.009 obtained using the lifetime technique. At the lowest thrust condition, the pressure distribution shows a slight low pressure region near the leading edge of the blade. From this region the pressure gradually increases toward the trailing edge. For the next thrust condition, $\mathrm{C}_{\mathrm{T}}=0.005$, a low pressure region exists near the leading edge of the blade and extends spanwise nearly the entire width of the painted region. Moving chordwise toward the trailing edge, pressure increases sharply within a very short distance and then continues gradually increasing until the trailing edge. At a thrust coefficient of 0.007 , a low pressure region at the leading edge is again observed, but it is not as strong and is more diffuse than the one observed at the previous thrust condition. At the highest thrust setting, $\mathrm{C}_{\mathrm{T}}=0.009$, the pressure distribution has a very strong and well defined low pressure region. Similar to the $\mathrm{C}_{\mathrm{T}}=$ 0.005 case, the low pressure region extends the entire span of the painted region. Additionally, it appears to turn the corner and exist for a short chordwise distance at the tip. Similar to the other cases, the pressure gradually increases chordwise to the trailing edge, after the sharp peak. 


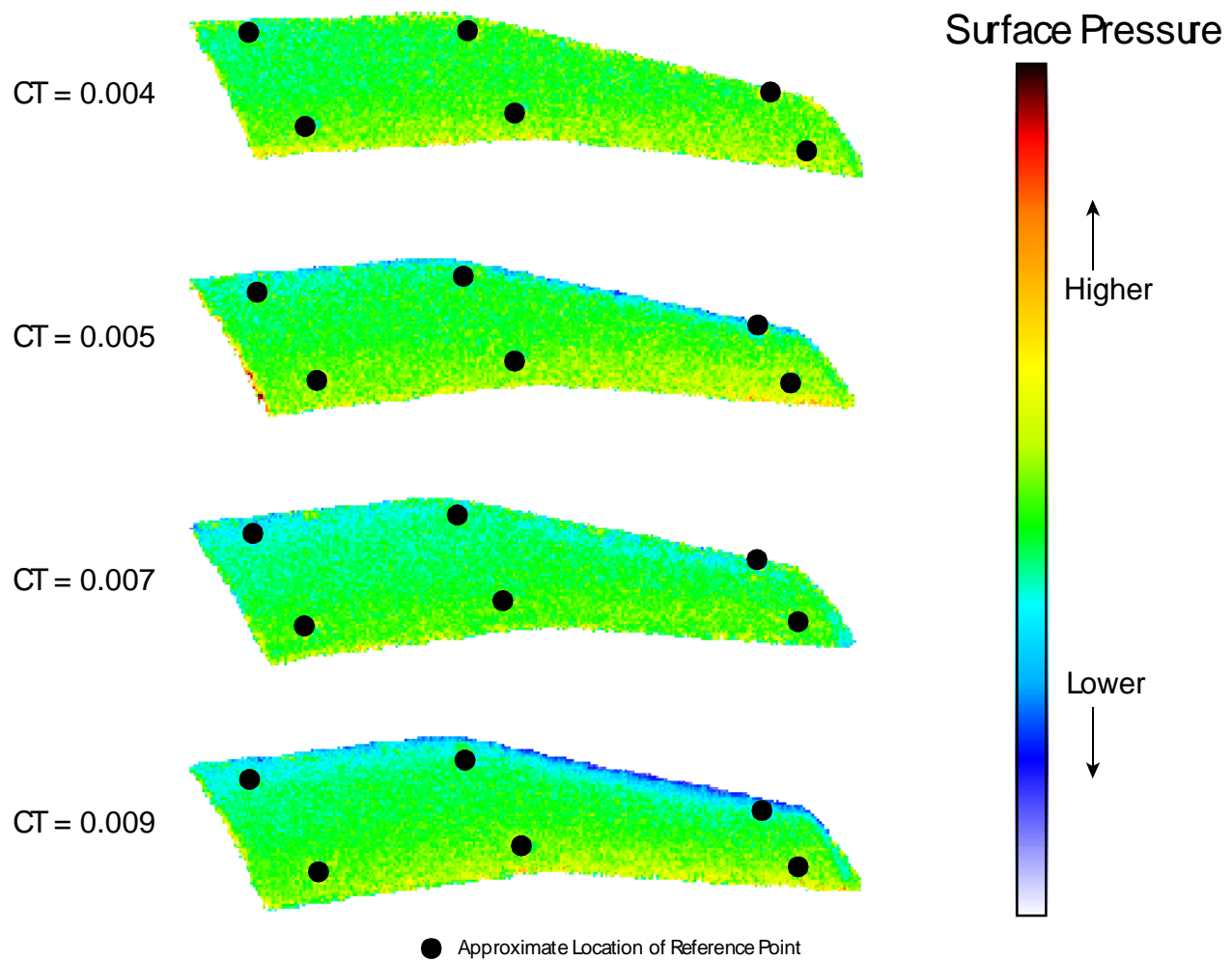

Figure 4. Blade 1 during a thrust sweep

\section{General Remarks}

It should be noted that the data shown are two-dimensional images of the blade. Due to the combination of the observation, collective, blade twist, flapping and lead-lag angles, the entire upper surface is not visible to the camera at all conditions. The combined effect of these angles changes across test conditions. This is illustrated by examining the location of the registration marks at the leading edge. The front registration marks on the leading edge are about 0.25 ” from the leading edge as shown in Fig. 3. This corresponds approximately to $\mathrm{x} / \mathrm{c}$ of 0.1 . The registration marks shown in the data, however, are very near to or at the apparent leading edge, depending on the thrust condition. At the lowest thrust condition the leading edge registration marks, especially at the tip, are clearly on the apparent leading edge (Fig. 5). In general, at higher thrust conditions, more of the upper surface is visible. This is illustrated by the registration marks "moving back" from the leading edge, and is believed to be primarily the result of the increased rotor collective. The increase in collective tilts the blade back (toward the camera), resulting in more exposure of the upper surface to the camera. The net result is that there is a fair degree of uncertainty in how much data is available forward of $\mathrm{x} / \mathrm{c} \approx 0.1$, especially at the low thrust conditions. 


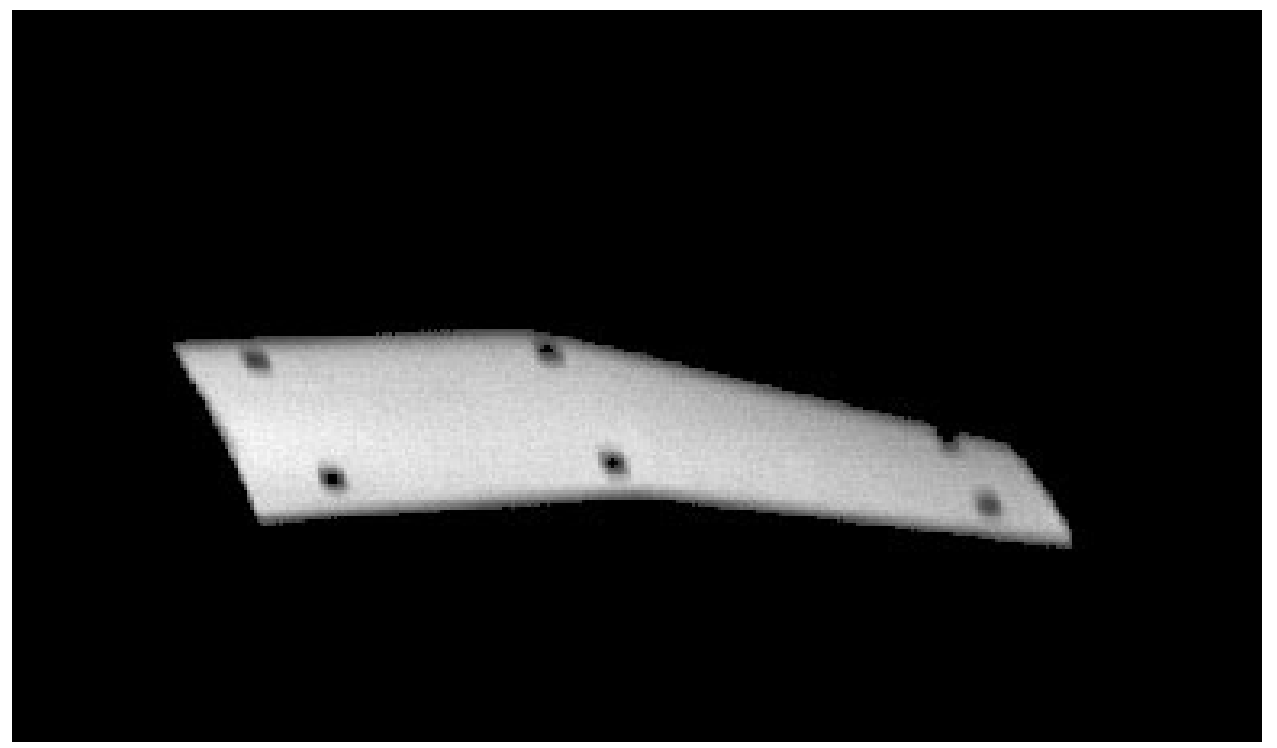

Figure 5. Typical raw lifetime image, $C_{T}=0.004$. Note: registration marks

\section{Leading Edge Low Pressure Region}

Perhaps the most interesting features observed in the data are the low pressure regions at the leading edge of the blade. Since the blade was not pressure instrumented for this proof of concept test, it is difficult to definitively know whether the low pressure region is the suction peak or an artifact of the measurement technique. Lower pressure corresponds to increased brightness; because of this any external light source contaminating the image would manifest itself as a low pressure region. One possibility is self illumination ${ }^{23-27}$. This occurs when the surface geometry results in the emission from a painted surface hitting another painted surface before being collected by the camera. In this case, the second surface appears to have a higher intensity than it really has. With the geometry of the blade, however, self illumination should not exist. Another possibility is glare from the excitation source. Even with a band-pass filter to block the excitation wavelength, some excitation photons may make it to the CCD since the filter is not $100 \%$ efficient. Since PSP is fundamentally a low light technique, with sufficient glare, enough photons from the excitation source may make it to the CCD to contaminate the image.

Most glare can be eliminated with careful placement of the camera and LEDs. Glare needs to be considered for each data point taken, however, since the angles between the camera, LEDs and blades change slightly at each condition. The raw images are carefully inspected to look for glare. An example of an image pair used to obtain pressure distributions is shown in Figure 6. Gate 2 is the data image and is taken when the paint is relaxing from its excited state. There are two bright regions marked A' and B'. These bright regions cannot be due to glare since the LEDs are off. They are either due to luminescence of the paint or are the result of some other light source. There was slight infiltration of daylight through the open louvers of the RTC, but it was dark enough that flashlights were required to move around safely. Background images were subtracted and should have removed the effect of any illumination by sources other than luminescence. The reference image, Gate 1, is where evidence of glare would be found. In Gate 1 there is only one bright region, Region A. This region needs to be treated with caution since glare would appear as a bright region in the data. However, since A is in the same physical location as A' and A' is from a condition where glare is not possible, the bright spot on the tip is likely due to inhomogeneties in the paint. Thus, it is unlikely that the leading edge low pressure region is the result of glare. It should be noted that inhomogeneties in the paint as well as in lighting are compensated for in the ratioing of the images. 


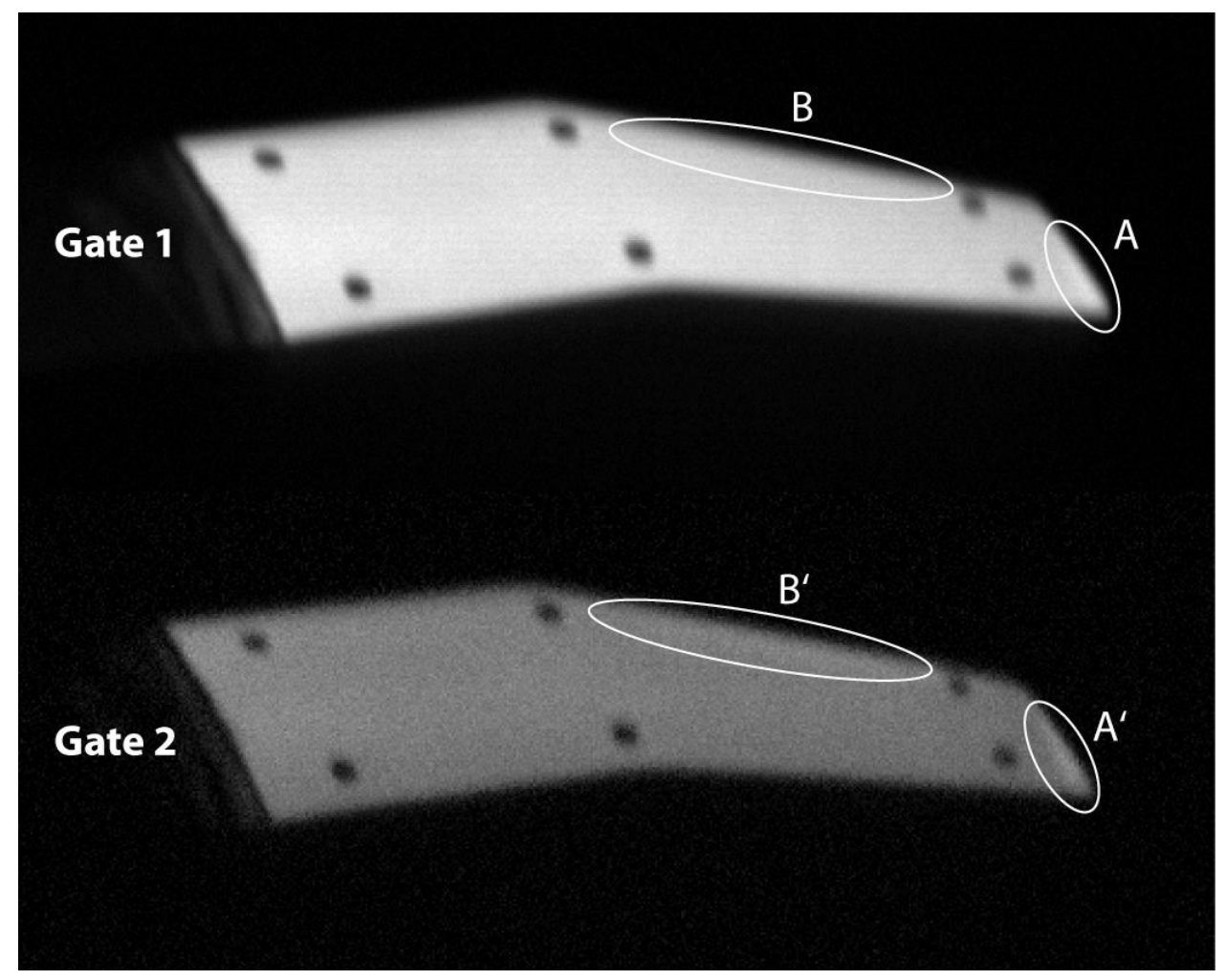

Figure 6. Typical raw image pair, $C_{T}=0.009$. Gate 1 is the reference image. Gate 2 is the data image.

The local $\alpha$ of the tip was calculated using Momentum Theory to estimate the induced velocity. The range of $\alpha$ for the conditions tested are shown in Table 4.

\begin{tabular}{|c|c|}
\hline $\begin{array}{c}\text { Thrust } \\
\text { Coefficient }\end{array}$ & $\begin{array}{c}\text { Estimated Local } \alpha \\
\text { r/R }=0.92 \text { to } 1.0\end{array}$ \\
\hline 0.004 & $0.6^{\circ}$ \\
\hline 0.005 & $2.1^{\circ}$ \\
\hline 0.007 & $3.7^{\circ}$ \\
\hline 0.009 & $5.3^{\circ}$ \\
\hline
\end{tabular}

Table 4. Blade Tip Local $\alpha, M_{\text {Tip }}=0.65$

The local $\alpha$ was then used to compare the PSP measured pressure distributions with experimentally measured airfoil data at similar conditions. The experimentally measured airfoil pressure distributions by Flemming ${ }^{28}$ are shown in Fig. 7. Flemming's data shows a broad low pressure region centered at a $\mathrm{x} / \mathrm{c}$ of 0.10 . As mentioned earlier, due to the observation and other angles, it is unknown how much of the upper surface is blocked from view. There is some variation in this dimension since the marks were drawn on the blades by hand. The PSP data from a $\mathrm{C}_{\mathrm{T}}$ of 0.004 shows a weak and broad low pressure region near the leading edge, in the approximate location of that seen in the airfoil data. Likewise at the highest thrust coefficient examined, the low pressure region is in the approximate location of the suction peak as measured by Flemming at an $\alpha$ of $6.3^{\circ}$. The $\mathrm{C}_{\mathrm{T}}=0.007$ case is the condition of most interest. In this condition, a strong low pressure region is not observed in the PSP data. Looking at the airfoil data, the $\alpha$ that is closest to this case, $\alpha=3.3^{\circ}$, shows the suction peak forming at approximately $6 \%$ of the chord. Since it is unclear how much of the leading edge is visible in the PSP data, it is possible that the suction peak on the blade is too far forward to be observed. Evidence of a weaker low pressure region is observed and is consistent with behavior of the pressures behind the suction peak observed in Flemming's measurements. The behavior of the low pressure region in the PSP data appears to be consistent with the experimentally measured airfoil pressure distributions. It is quite possible that the low pressure region observed in the PSP data is actually the blade's suction peak. 


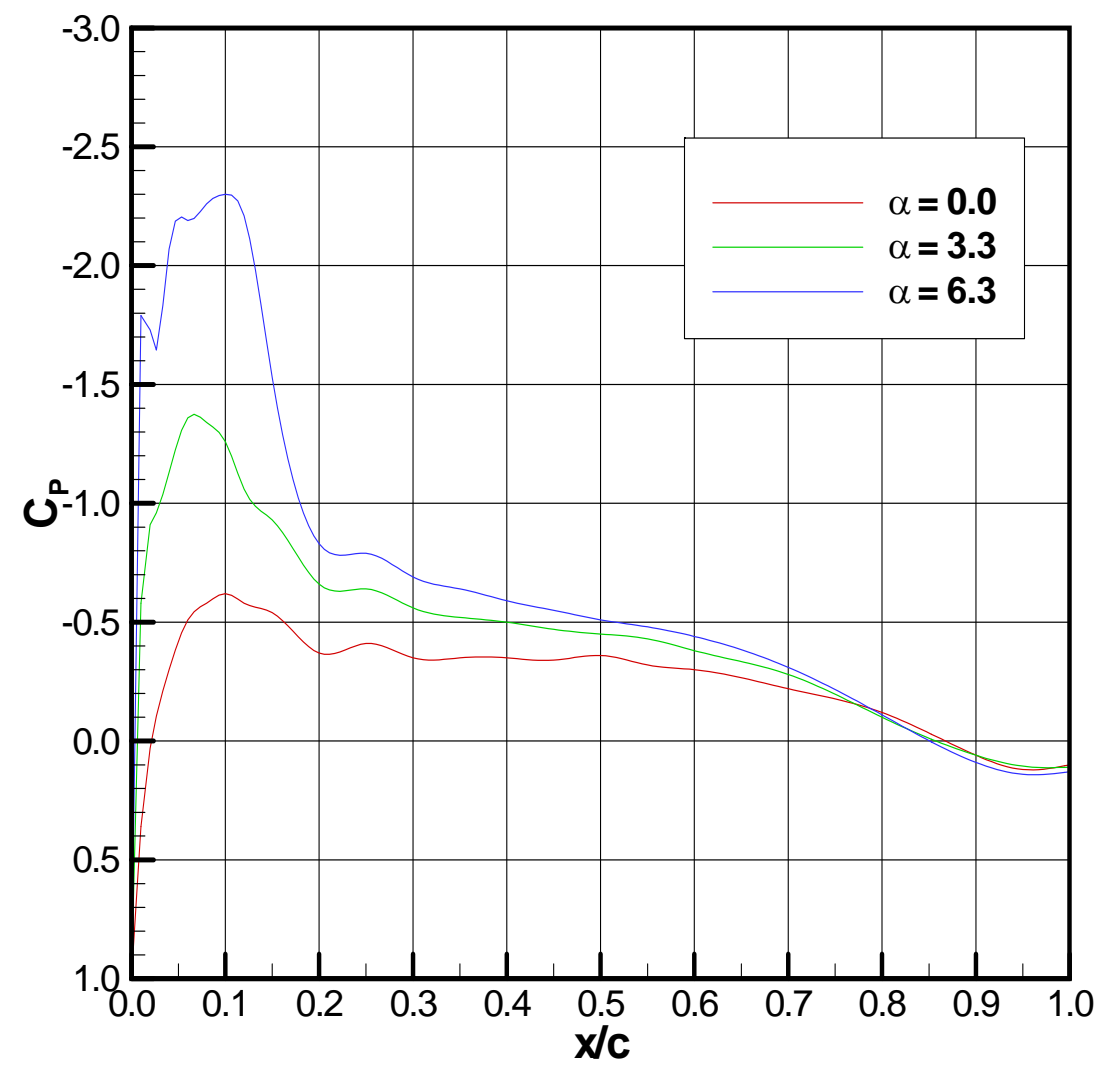

Figure 7. Experimentally Measured Airfoil Pressure Distributions, $\mathrm{M}=\mathbf{0 . 6 0}$ (Flemming ${ }^{28}$ )

\section{E. Blade-to-Blade Variation}

The pressure distributions of all five blades at $\mathrm{C}_{\mathrm{T}}=0.009$ are shown in Fig. 8 . The blades have a very strong low pressure region near the leading edge that is, based on the previous discussion, likely the suction peak. Following that is a strong positive gradient followed by a gradual increase in pressure to the trailing edge. The small high pressure region on the inboard edge of the painted region is an artifact of the markings used to differentiate the blades. The suction peak is very apparent on all of the blades except for Blade 2 .

Closer examination of the other blades show variations in the strength of the suction peak. To a certain degree this is to be expected, due to slight manufacturing differences. During the experimental setup it was noted that these blades did not track well. At a given condition, even after adjusting individual blade pitch, the blades did not all fly in the same plane. During typical rotor experiments, the track of the rotor is adjusted so that all of the blade tips are within one to two blade tip thicknesses of each other. In this experiment it was not possible to track the blades to better than three to four blade tips thicknesses.

For all of the thrust conditions tested, Blade 2 exhibited pressure distributions significantly different than the other four blades. It seems unlikely that the blade was flying in an orientation that prevented the observation of the suction peak at all conditions. Thus, either Blade 2 is a maverick blade and has an unusual pressure distribution or there was an issue with the paint on Blade 2. Of all the blades, Blade 2 was the most difficult to track. Cursory inspection of the paint during the test did not reveal anything grossly wrong with the paint. Unfortunately, without pressure measurements to compare to the PSP data, we cannot definitively determine why Blade 2 behaved differently than the rest. 


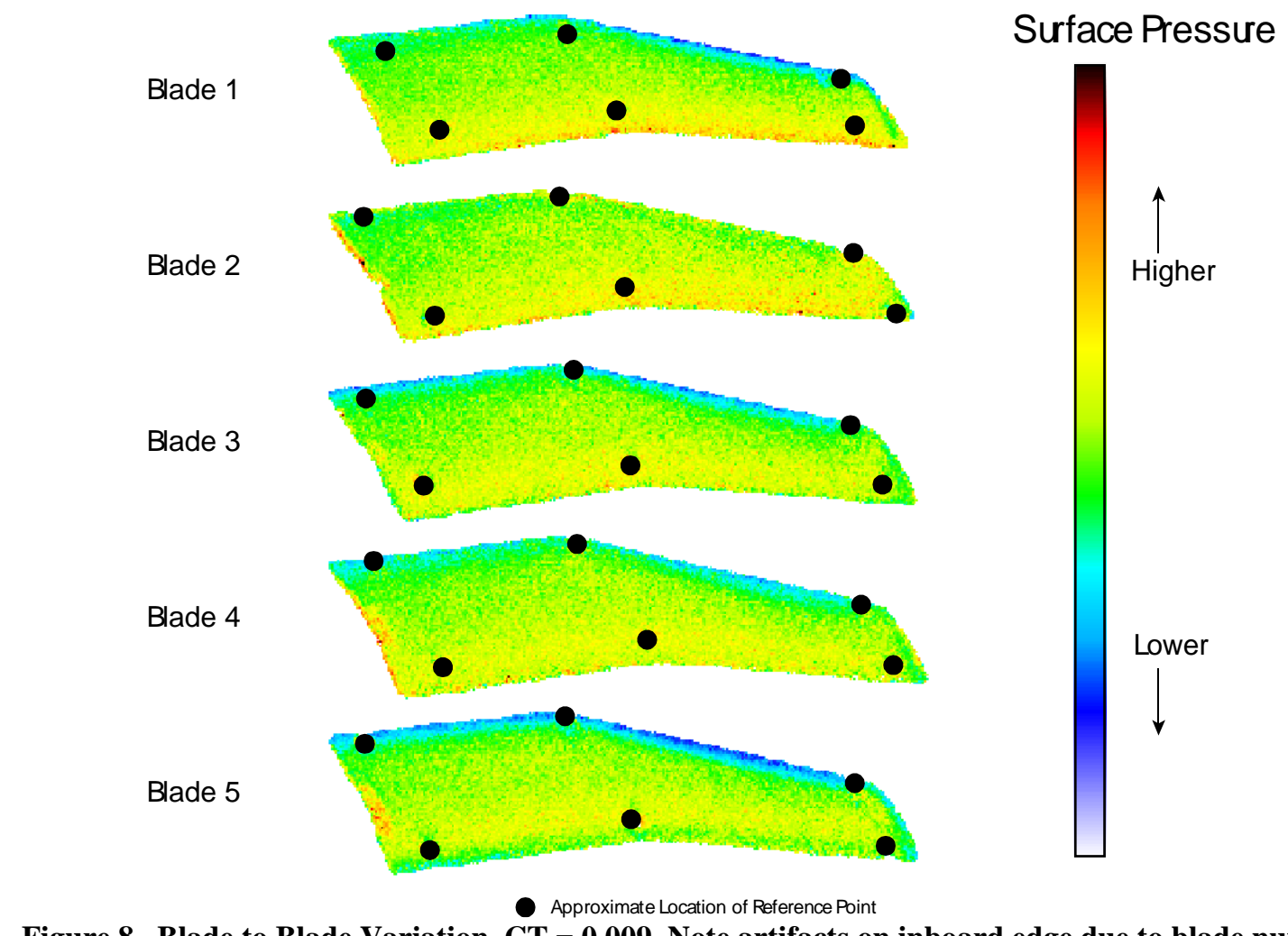

Figure 8. Blade to Blade Variation, $\mathrm{CT}=\mathbf{0 . 0 0 9}$. Note artifacts on inboard edge due to blade numbering

\section{Summary}

This paper discusses a proof of concept test conducted to evaluate the suitability of using PSP to measure the pressure distribution on rotor blades. These tests were conducted on a five-bladed hub in hover for low to moderate thrust conditions $\left(\mathrm{C}_{\mathrm{T}}=0.004\right.$ to 0.009$)$. Intensity and lifetime PSP measurement techniques were attempted using Ruthenium bathophenanthroline. Since this was a proof of concept test certain limitations were accepted. The primary limitation was the inability to accurately account for temperature effects on the PSP. Even though quantitative pressure measurements were made, it was not possible to accurately reference the pressure measurements absolutely.

Measurements of the blades during a thrust sweep show an apparent low pressure region forming with increasing thrust at the leading edge of the blade. Comparison of this data with airfoil data at similar conditions suggest that the observed low pressure region is the suction peak of the blade. Additional tests were conducted to examine the blade to blade variation in pressure distribution. Four of the five blades were found to have similar pressure distributions. In this set of four blades, the observed differences are believed to be the result of manufacturing differences. Blade 2 was found to have a significantly different pressure distribution than the other blades for all thrust conditions. At this point it is not possible to say whether Blade 2 is a maverick blade or if there were issues with the paint on Blade 2 .

\section{Recommendations and Future Plans}

The data obtained in this proof of concept test suggest that there is strong potential for using PSP as a new technique for measuring pressure distributions on rotor blades. Developing PSP as a feasible measurement technique could advance current understanding of the fundamental physics of the flow in the tip region. Further research is recommended, collecting measurements that do not experience the same limitations encountered in this work. A set of rotor blades with chordwise pressure tap arrays is being designed. The pressure taps will enable in situ calibration of the PSP so that temperature sensitivity can be accounted for. Additionally, these pressure taps will also provide reference pressure measurements for direct comparison with the PSP measurements. In addition to 
working with the new rotor blades, other future plans include mapping PSP data onto the blade surface and using a variety of camera angles to capture pressure distributions on the leading edge of the blade.

\section{Acknowledgments}

The authors would like to acknowledge W. Derry Mace, Curtis Fossum, Andrew Harrison, Bradley Leighty and William Lipford for their assistance during these measurements. Discussions with Wayne Mantay and Kevin Noonan were also greatly appreciated.

\section{References}

${ }^{1}$ Lorber, P.F., Stauter, R.C. and Landgrebe, A.J., “A Comprehensive Hover Test of the Airloads and Airflow of an Extensively Instrumented Model Helicopter Rotor,” Proceedings of the 45th Annual Forum of the American Helicopter Society, May 1989.

${ }^{2}$ Lal, M.K., Liou, S.G., Pierce, G.A. and Komerath, N.M., "Measurements Around a Rotor Blade Excited In Pitch, Part 2: Unsteady Surface Pressure,” Journal of the American Helicopter Society, April 1994, pp 13 - 20.

${ }^{3}$ Gorton, S.A, Poling, D.R., and Dadone, L., "Laser Velocimetry and Blade Pressure Measurements of a Blade-Vortex Interaction,” Journal of the American Helicopter Society, April 1995.

${ }^{4}$ Lorber, P.F., “Aerodynamic Results of a Pressure-Instrumented Model Rotor Test at the DNW”, Proceedings of the 46th Annual Forum of the American Helicopter Society, May 1990.

${ }^{5}$ Shimbo, Y., Asai, K., and Komatsu, N., "Pressure sensitive paint application at large production wind tunnels," International Congress of Aeronautical Sciences, 22nd, Harrogate, United Kingdom, Aug. 27-Sept. 1, 2000.

${ }^{6}$ Mebarki, Y., "Pressure sensitive paints - From laboratory to wind tunnel," International Congress of Aeronautical Sciences, 22nd, Harrogate, United Kingdom, Aug. 27-Sept. 1, 2000.

${ }^{7}$ Mebarki, Y., "Pressure sensitive paints: Application in wind tunnels (Peintures Sensibles a la Pression: Application en Soufflerie Aerodynamique)," Vol. NASA No. 19990026234; ONERA-NT-1998-6, 1998.

${ }^{8}$ Morris, M.J., Donovan, J.F., and Kegelman, J.T., "Aerodynamic applications of pressure sensitive paint," AIAA Journal, Vol. 31, No. 3, 1993.

${ }^{9}$ Bencic, T.J., "Rotating pressure and temperature measurements on scale-model fans using luminescent paints," AIAA/ASME/SAE/ASEE Joint Propulsion Conference \& Exhibit, 34th, Cleveland, OH, July 13-15, 1998, Vol. AIAA Paper 983452, 1998.

${ }^{10}$ Gregory, J.W., Sakaue, H., and Sullivan, J.P., "Unsteady pressure measurements in turbomachinery using porous pressure sensitive paint,” AIAA-2002-84, 40 ${ }^{\text {th }}$ AIAA Aerospace Sciences Meeting and Exhibit, Reno, NV, Jan. 14-17, 2002.

${ }^{11}$ Sakaue, H., Gregory, J.W., and Sullivan, J.P., "Pressure sensitive paint for unsteady flow fields," AIAA, Aerospace Sciences Meeting and Exhibit, 39th, Reno, NV, Jan. 8-11, 2001, Vol. AIAA Paper 2001-0554, 2001.

${ }^{12}$ Bosnyakov, S., Bykov, A., and Coulech, V., "Blade deformation and PSP measurements on the large scale rotor by video metric system," ICIASF '97 - International Congress on Instrumentation in Aerospace Simulation Facilities, 17th, Pacific Grove, CA, Sept. 29-Oct. 2, 1997, Record (A98-18712 03-35), Piscataway, NJ, Institute of Electrical and Electronics Engineers, Inc., 1997, p. 95-104, Piscataway, NJ: Institute of Electrical and Electronics Engineers, Inc., 1997, pp. 95.

${ }^{13}$ Koulesh, V.P., Mosharov, V.E., and Radchenko, V.N., Improvement of Fast Binary Pressure-Sensitive Paint Technology for Helicopter Rotor Blade Investigations, Vol. AD-A395378; ARO-39806.1-EG, 2000.

${ }^{14}$ Sakaue, H., Matsumura, S., Schneider, S., and Sullivan, J., “Anodized Aluminum Pressure Sensitive Paint for Short Duration Testing ,”AIAA-2002-2908, $22^{\text {nd }}$ AIAA Aerodynamic Measurement Technology and Ground Testing Conference, St. Louis, Missouri, June 24-26, 2002.

${ }^{15}$ Sakaue, H., and Sullivan, J.P., "Fast response time characteristics of anodized aluminum pressure sensitive paint,” AIAA2000-506, 38 ${ }^{\text {th }}$ AIAA Aerospace Sciences Meeting and Exhibit, Reno, NV, Jan. 10-13, 2000.

${ }^{16}$ Sakaue, H., Gregory, J.W., Sullivan, J.P., and Raghu, R., "Porous Pressure-Sensitive Paint for Characterizing Unsteady Flowfields,” AIAA Journal, Vol.40, No.6, 2002, pp. 1094 - 1098.

${ }^{17}$ Sakaue, H., and Sullivan, J.P., “Time Response of Anodized Aluminum Pressure-Sensitive Paint,” AIAA Journal, Vol.39, No.10, 2001, pp. 1944 - 1949.

${ }^{18}$ Hamner, M.P., "Demystifying luminescent paint technology - A guide for non-developers," AIAA Fluid Dynamics Conference \& Exhibit, 31st, Anaheim, CA, June 11-14, 2001, Vol. AIAA Paper 2001-2981, 2001.

${ }^{19}$ Liu, T., and Sullivan, J.P., "Pressure and Temperature Sensitive Paints (Experimental Fluid Dynamics)," Springer-Verlag, Berlin, 2004.

${ }^{20}$ Phelps, A.E., Berry, J.D., Description of the U.S. Army Small-Scale 2-Meter Rotor Test System, NASA TM-87762, AVSCOM TM 86-B-4, 1987.

${ }^{21}$ Bencic, T., "Status Update: Dynamic Calibration Rigs at NASA Glenn,” 9th PSP Workshop March, 7-11, 2002, Washington DC.

${ }^{22}$ Watkins, A.N., Jordan, J.D., Leighty, B.D., Ingram, J.L., and Oglesby, D.M., "Development of Next Generation Lifetime PSP Imaging Systems," Proceedings of the 20th International Congress on Instrumentation in Aerospace Facilities, 2003. 
${ }^{23}$ Ruyten, W.M., "Correction of Self-illumination and Spectral Leakage Effects in Luminescent-Painr Measurements," Applied Optics, Vol. 36, No. 14, 1997, pp. 3079-3085.

${ }^{24}$ Ruyten, W.M., "Self-Illumination Calibration Technique for Luminescent Paint Measurements," Review of Scientific Instrumentation, Vol. 69, No. 9, 1997, pp. 3452-3457.

${ }^{25}$ Ruyten, W.M, and Fisher, C., "Effects of Reflected Light in Luminescent Paint Measurements," AIAA Journal, Vol. 39, No. 8, 2001, pp. 1587-1592.

${ }^{26}$ Ruyten, W.M., "Reconstruction of the Net Emission Distribution fro the Total Radiance Distribution on a Reflecting Surface," Journal of the Optical Society of America A, Vol. 18, No. 1, pp. 216-223.

${ }^{27}$ Le Sant, Y., "Overview of the Self-Illumination Effect Applied to Pressure Sensitive Paint Applications," Proceedings of the 19th International Congress on Instrumentation in Aerospace Simulation Facilities, Cleveland, Ohio, August 27-30, 2001.

${ }^{28}$ Flemming, R.J., "An Experimental Evaluation of Advanced Rotorcraft Airfoils in the NASA Ames Eleven-foot Transonic Wind Tunnel,” NASA CR-166587, 1984. 